\title{
miR-193b acts as a cisplatin sensitizer via the caspase-3- dependent pathway in HCC chemotherapy
}

\author{
WENJUN YIN $^{1 *}$, YUEHUA NIE $^{2 *}$, ZHIWEI ZHANG $^{1}$, LIMING XIE $^{3}$ and XIUSHENG HE ${ }^{1}$ \\ ${ }^{1}$ Cancer Research Institute of Medical College, University of Southern China, \\ University Key Laboratory of Cancer Cellular and Molecular Pathology in Hunan Province; \\ Departments of ${ }^{2}$ Radiation Oncology and ${ }^{3}$ Chemotherapy Oncology, \\ The First Affiliated Hospital, University of South China, Hengyang, Hunan 421001, P.R. China
}

Received March 11, 2015; Accepted April 30, 2015

DOI: $10.3892 /$ or.2015.3996

\begin{abstract}
Mounting evidence suggests that microRNAs (miRNAs) play important roles in the development of cancer by targeting expression of tumor-related genes. In the present study, downregulation of miR-193b was observed in hepatocellular carcinoma (HCC) tissues and HCC cell lines by quantitative RT-PCR analyses, suggesting that miR-193b is a tumor-suppressor in HCC. More importantly, miR-193b significantly enhanced the cytotoxicity of cisplatin in HepG2 cells by targeting Mcl-1. Knockdown of the Mcl-1 gene by specific siRNA exhibited a function similar to miR-193b on sensitizing HepG2 cells to cisplatin-inducing cytotoxicity. Furthermore, the miR-193b-induced sensitization of HepG2 cells to cisplatin cytotoxicity was abolished by the transfection of Mcl-1 expression plasmid that lacked the 3'-untranslated region (3'-UTR). In addition, activation of caspase-3 was needed for sensitization by $\mathrm{miR}-193 \mathrm{~b}$ to cisplatin-mediated cell death. Thus, the present study revealed the downregulation of miR-193b in HCC cells and illustrated a synergistic effect on cisplatin-induced apoptosis by targeting Mcl-1.
\end{abstract}

\section{Introduction}

Hepatocellular carcinoma (HCC) is one of the most prevalent types of cancer and the leading cause of human mortality worldwide (1). Aberrant gene expression and somatic mutations induced by genotoxic agents that cause DNA damage remain one main process leading to the development of HCC (2). Most patients are diagnosed at a late stage and thus miss

Correspondence to: Dr Xiusheng He, Cancer Research Institute of Medical College, University of Southern China, University Key Laboratory of Cancer Cellular and Molecular Pathology in Hunan Province, 28 Changsheng Road, Hengyang, Hunan 421001, P.R. China E-mail: hunanxiushenghe@163.com

*Contributed equally

Key words: hepatocellular carcinoma, miR-193b, Mcl-1, cisplatin, apoptosis, caspase-3 the most optimal period for effective treatment. Systematic chemotherapy plays a crucial role in HCC treatment especially for patients with terminally staged tumors (3). Cisplatin is a widely used chemotherapeutic agent for the treatment of HCC. However, a high dose of cisplatin causes serious side effects and also kills normal cells, and the long-term side effects of cisplatin, which are carcinogenic can cause secondary cancers (4).

microRNAs (miRNAs) belong to a class of small, endogenous, non-coding, single-stranded RNAs, which are $\sim 22$ nucleotides in length. Growing evidence indicates that miRNAs functionally repress target proteins by pairing with the 3'-untranslated region (3'-UTR) of specific target mRNAs, inducing mRNA degradation or translational repression (5-7). Studies have demonstrated that miRNAs are involved in many biological processes such as cell proliferation, differentiation, development and apoptosis $(8,9)$. miRNAs also play important roles in the development of cancer, and may act as either oncogenic molecules or tumor suppressors by regulating respective target genes $(10,11)$. However, the role of miRNAs in cancer chemotherapy remains largely unknown.

Herein, we provided evidence showing the absence of miR-193b expression in HCC cells. Furthermore, we demonstrated that miR-193b significantly enhanced the antitumor effect of cisplatin in HCC therapy by targeting Mcl-1, which is an anti-apoptotic Bcl-2 family member (12). Furthermore, the activation of caspase-3 was required for the sensitization of HCC cells by miR-193b to cisplatin-mediated cell death.

\section{Materials and methods}

Reagents. Cisplatin, zVAD-fmk, 3-(4,5-dimethylthiazol-2-yl)-2,5-diphenyltetrazolium bromide (MTT), dimethyl sulfoxide (DMSO) and the Annexin V-FITC Apoptosis Detection kit were obtained from Sigma-Aldrich (St. Louis, MO, USA). Antibodies for rabbit anti-human Mcl-1, rabbit anti-human caspase-3, rabbit anti-human poly-ADP-ribose polymerase (PARP) and rabbit anti-human $\beta$-actin were purchased from Cell Signaling Technology (Danvers, MA, USA). miR-193b mimics, Mcl-1 siRNA and the negative control oligonucleotide (NCO) were purchased from GenePharma Company (China). 
Clinical specimens. Forty pairs of HCC tumor and corresponding adjacent non-tumor liver tissues were obtained from patients who underwent hepatic tumor resection at the First Affiliated Hospital, University of South China from May 2011 to March 2014. The use of clinical tissues for this study was approved by the hospital's Protection of Human Subjects Committee.

Cell culture and transfection. Normal hepatic cell line L02 and HCC cell lines including Huh7, HepG2 and PLC were obtained from the Institute of Biochemistry and Cell Biology, Chinese Academy of Sciences (Shanghai, China) and cultured in Dulbecco's modified Eagle's medium (DMEM) basic medium with $10 \%$ fetal bovine serum (FBS) (both from Gibco, Carlsbad, CA, USA) at $37^{\circ} \mathrm{C}$ in a humidified $5 \% \mathrm{CO}_{2}$ incubator. HepG2 cells were transfected with the miR-193b mimic (AACUGGCCCUCAAAGUCCCGCU), NCO (GGC CCUAAAGAACUUCCUCCCG), Mcl-1 siRNA (AAGUA UCACAGACGUUCUUU) and the recombinant pMIR or recombinant pcDNA3.1 using Lipofectamine 2000 (Invitrogen, Carlsbad, CA, USA) according to the protocols of the manufacturer.

Quantitative real-time polymerase chain reaction ( $R T-q P C R)$. Total RNA was extracted from the patient tissues or the HepG2 cells using TRIzol (Invitrogen), and the cDNA was synthesized using M-MLV reverse transcriptase (Invitrogen) following the manufacturer's instructions. The reverse transcription of miR-193b was performed by stem-loop RT-qPCR method (13) and the RT-primer sequences are as follows: 5'-CTCAACTGGTGTCGTGGAGTCGGCAATTCAGTTG AGAGCGGGAC-3'. The expression of mature miR-193b was quantified by real-time PCR using SYBR-Green (Takara, Japan) according to the manufacturer's instructions. Quantification of U6 was used to normalize the miRNA expression level using the $2^{-\triangle \Delta C T}$ method (14). U6 forward primer was 5'-CTCGCTTCGGCAGCACA3'. The expression of Mcl-1 mRNA was also determined by qPCR and the GAPDH mRNA was taken as the internal control. The primer sequences are as follows: Mcl-1 forward, 5'-GGCTAAACAC TTGAAGACCA-3' and reverse, 5'-TGGAAGAACTCCACA AACC-3'; GAPDH forward, 5'-CACTCCTCCACCTTTGA-3' and reverse, 5'-CCACCACCCTGTTGCTG-3'.

Plasmid construction. To construct the pMIR-Mcl-1 3'-UTR-WT plasmid, a wild-type 3'-UTR segment of human Mcl-1 mRNA (927-2348 nt, GenBank accession no. NM_001197320) containing the putative miR-193b binding sequence was amplified by PCR using the following primers: forward, 5'-AGGGCAAGAGGATTAT-3' and reverse, 5'-CTGTAGAG GGAGCAGAA-3', and then cloned into the downstream of the firefly luciferase gene in the pMIR-REPORT $^{\mathrm{TM}}$ miRNA Expression Reporter Vector (Life Technologies, Carlsbad,CA, USA). pMIR-Mcl-1 3'-UTR-MUT, which carried the mutated sequence in the complementary site for the seed region of miR-193b (GGCCAGU to GGCGUGU) was generated using the Site-Directed Mutagenesis kit (Takara) based on the wild-type plasmid. The open reading frame of the Mcl-1 gene without 3'-UTR was amplified by PCR with the cDNA as template and cloned into the pcDNA3.1 vector (Invitrogen); the recombinant plasmid was named pcDNA3.1-Mcl-1.

Luciferase reporter assay. HepG2 cells were incubated in 48 -well plates. The cells were co-transfected with $50 \mathrm{pmol} / \mathrm{ml}$ of either miR-193b mimics or NCO plus $2 \mu \mathrm{g} / \mathrm{ml}$ of either pMIR-Mcl-1 3'-UTR-WT or pMIR-Mcl-1 3'-UTR-MUT plasmid, and $100 \mathrm{ng} / \mathrm{ml}$ of the Renilla luciferase pRL-TK vector (Promega, Madison, WI, USA). Cells were collected $24 \mathrm{~h}$ after transfection and analyzed using the Dual-Luciferase Reporter system (Promega) according to the manufacturer's instructions. Firefly luciferase activity was normalized to the Renilla luciferase activity.

Western blot analysis. Protein extracts were separated by 12.5\% SDS-PAGE and transferred onto PVDF membranes. This was followed by probing with rabbit primary antibodies against human Mcl-1 and $\beta$-actin. The membranes were then incubated with a horseradish peroxidase-conjugated secondary antibody or goat anti-rabbit IgG (Cell Signaling Technology). After washing, the proteins were visualized with an enhanced chemiluminescence detection kit (Pierce, Rockford, IL, USA).

Measurement of cell viability by MMT assay. HepG2 cells were seeded in triplicate in a 96 -well plate at a density of $3 \times 10^{3} /$ well. After incubation for $12 \mathrm{~h}$, the cells were transfected with miR-193b, Mcl-1 siRNA or NCO. After incubation for $24 \mathrm{~h}$, the HepG2 cells were treated with cisplatin for another $48 \mathrm{~h}$. MTT $(20 \mathrm{ml}, 5 \mathrm{mg} / \mathrm{ml})$ was added to each well and incubated for $4 \mathrm{~h}$. Medium was aspirated and $100 \mu \mathrm{l}$ DMSO was added to each well. Absorbance was read at OD 570/655 nm using the Bio-Rad Model 680 microplate reader (Bio-Rad, Hercules, CA, USA). Results are represented as the ratio between the various treatments and the negative control.

Apoptosis assay. HepG2 cells were transfected with miR-193b or NCO for $48 \mathrm{~h}$, and then cells were treated with cisplatin for another $48 \mathrm{~h}$. After treatment, the cells were incubated with Annexin V/propidium iodide (PI) for $15 \mathrm{~min}$ at room temperature according to the manufacturer's instructions and analyzed using flow cytometry (Becton Dickinson, San Jose, CA, USA).

Statistical analysis. Data are expressed as mean \pm SE. Student's t-test and ANOVA analysis were conducted with SPSS 14.0 software to assess the statistical significance between treatments. A $\mathrm{P}<0.05$ was considered to indicate a statistically significant difference.

\section{Results}

Downregulation of miR-193b in HCC patient tumor tissues and cell lines. The expression of miR-193b in 40 paired samples of clinical HCC tumor and adjacent normal liver tissues was determined using RT-qPCR assays. We found that miR-193b expression was significantly decreased in the HCC tumor tissues compared to this level in the adjacent normal liver tissues (Fig. 1A). To validate this finding, the expression of miR-193b was determined in liver cell lines. The results showed that the expression of miR-193b was substantially 

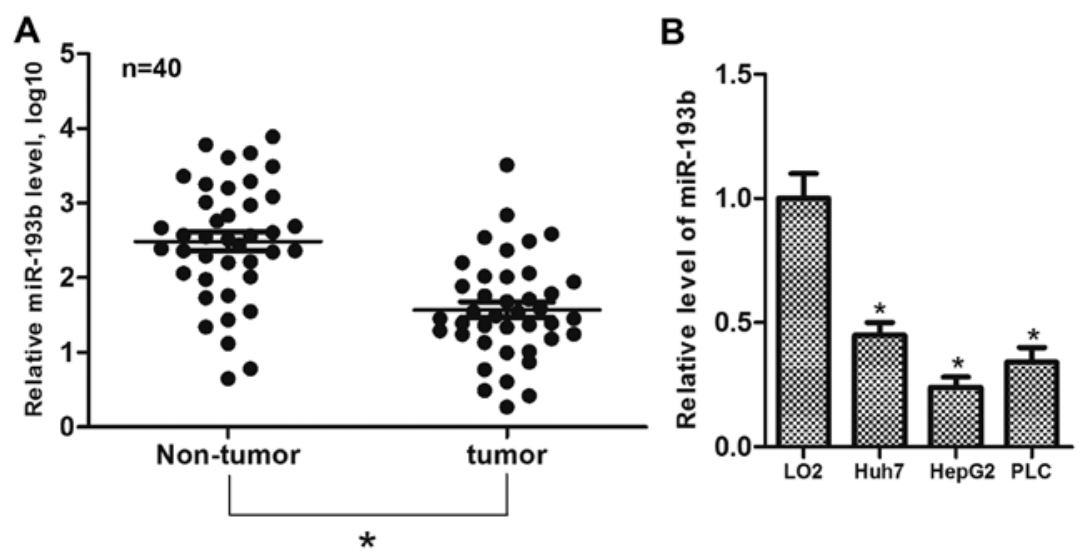

Figure 1. Analysis of miR-193b expression in human HCC tissues and cancer cell lines by RT-qPCR. (A) miR-193b expression in 40 paired HCC tumor and adjacent normal liver tissues. "P<0.05. (B) miR-193b expression in L02 and various HCC cell lines. ${ }^{\text {" }} \mathrm{P}<0.05$ vs. L02 cells. HCC, hepatocellular carcinoma.

A

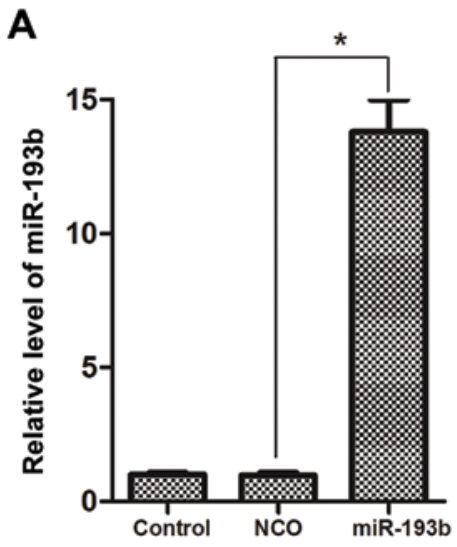

C

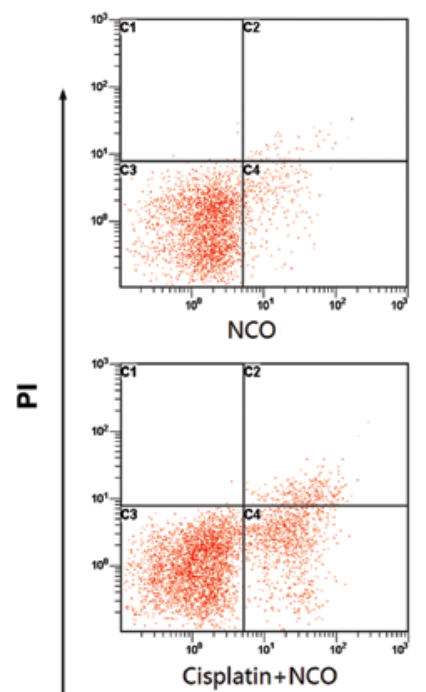

B
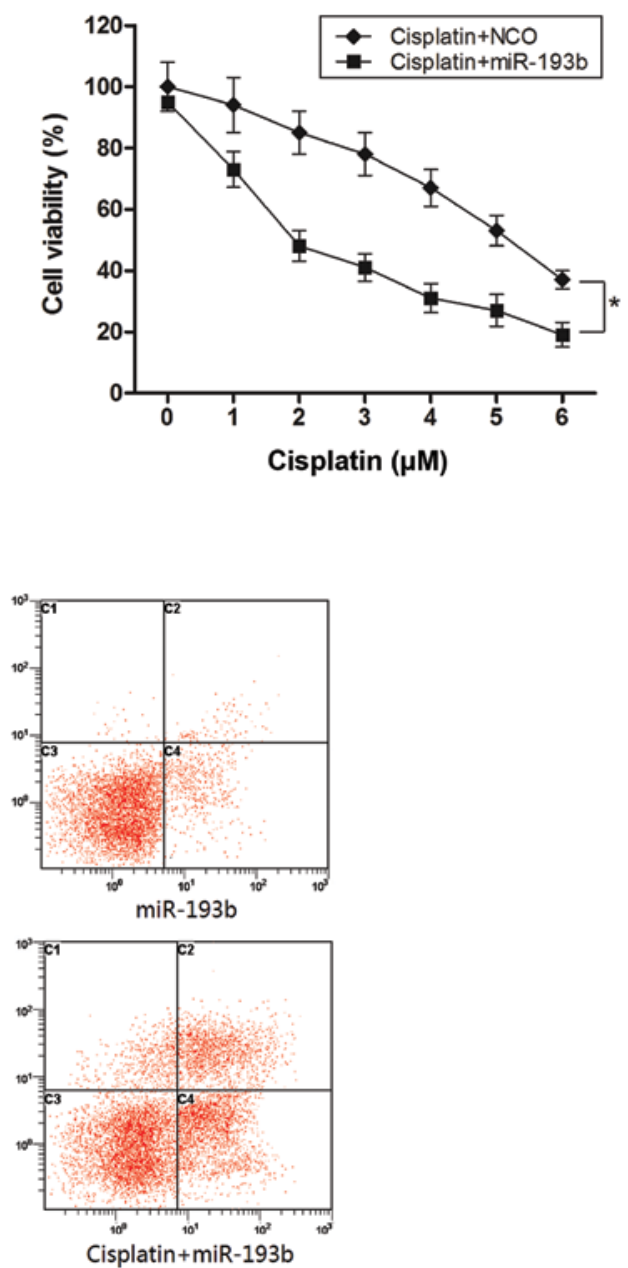

Annexin V-FITC

Figure 2. Overexpression of miR-193b enhances the cisplatin cytotoxicity in HepG2 cells. (A) qPCR analysis of HepG2 cells transfected with either miR-193b mimic or NCO $(50 \mathrm{pmol} / \mathrm{ml}),{ }^{*} \mathrm{P}<0.05$. (B) MTT assay was performed to evaluate the viability of HepG2 cells after treatment with different concentrations of cisplatin plus miR-193b mimic or NCO $(50 \mathrm{pmol} / \mathrm{ml}),{ }^{*} \mathrm{P}<0.05$. (C) Apoptotic rate of HepG2 cells was determined using Annexin V/PI staining following treatment of cisplatin $(2 \mu \mathrm{M})$ plus miR-193b mimic or $\mathrm{NCO}(50 \mathrm{pmol} / \mathrm{ml})$. NCO, negative control oligonucleotide; PI, propidium iodide.

lower in the HCC cell lines than that in the L02 cells (Fig. 1B). These results suggest that miR-193b may function as a tumor suppressor in HCC.
miR-193b sensitizes cisplatin-induced apoptosis in HepG2 cells. To explore the effect of miR-193b on cisplatin treatment in HCC, we used miR-193b mimics to increase the intracellular 
A

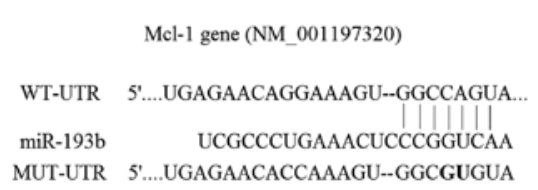

C

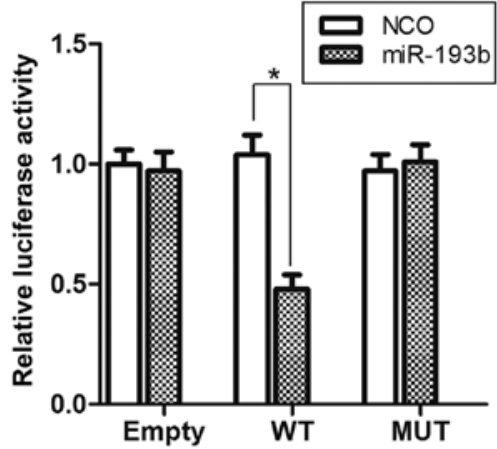

B

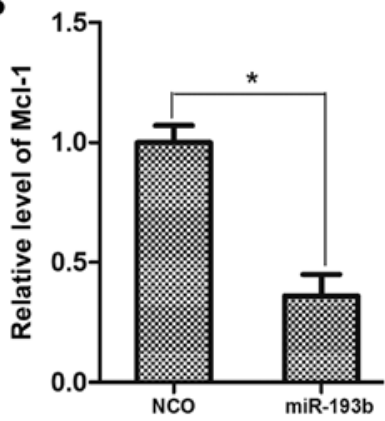

D
NCO miR-193b
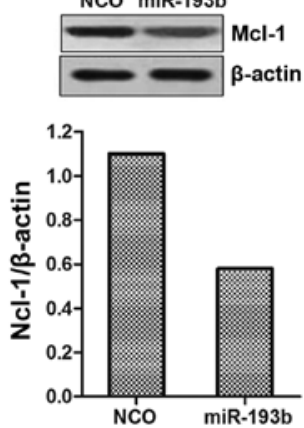
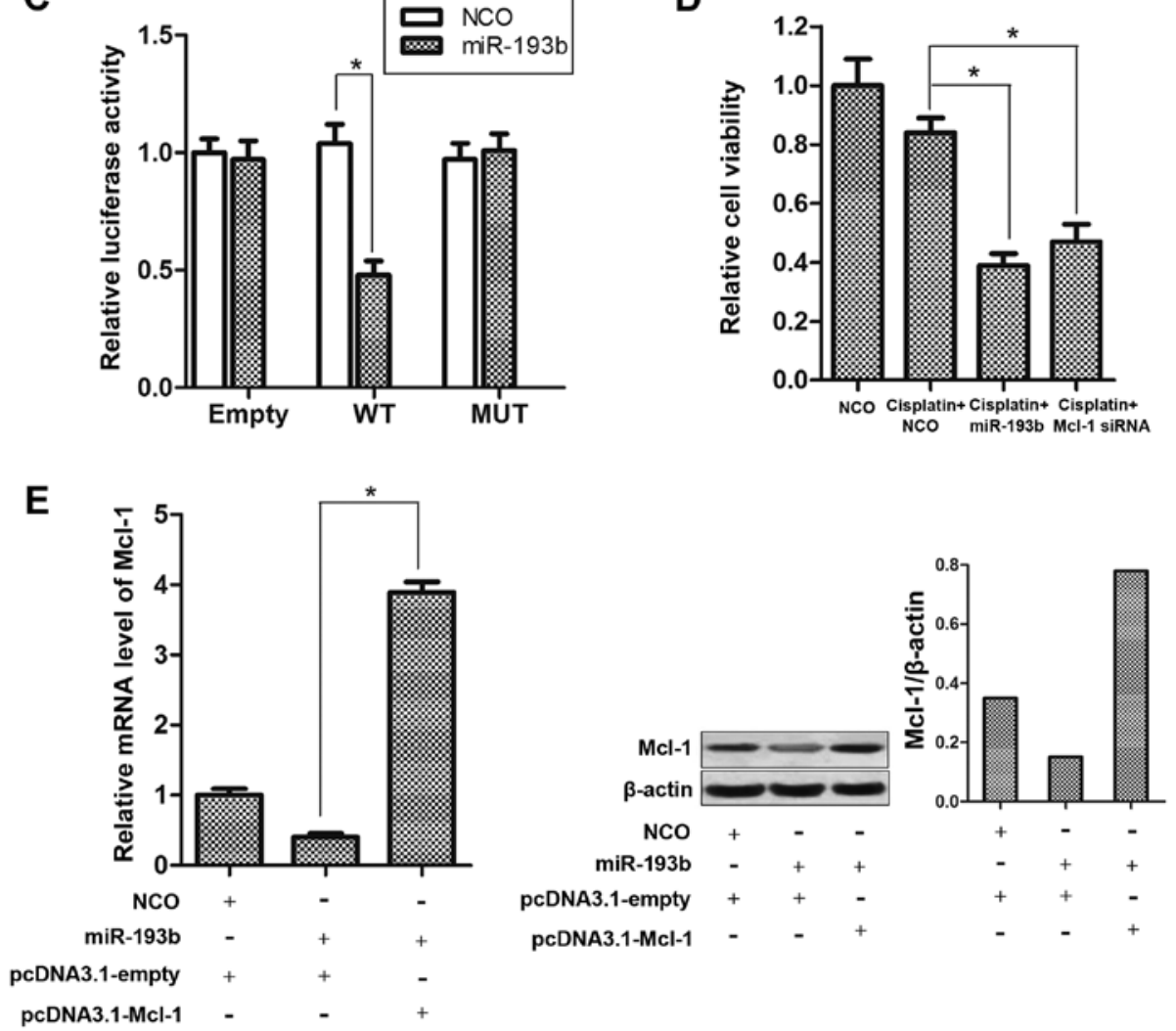

Figure 3. Mcl-1 is negatively regulated by miR-193b in HCC cells. (A) Putative miR-193b binding sequence in the 3'-UTR of Mcl-1 mRNA. Mutation was generated on the Mcl-1 3'-UTR sequence in the complementary site for the seed region of miR-193b. (B) Mcl-1 expression level was determined after miR-193b transfection by qPCR analysis and western blot analysis, ${ }^{*} \mathrm{P}<0.05$. (C) HepG2 cells were co-transfected with the pMIR reporter and miR-193b, and incubation was carried out for $24 \mathrm{~h}$. Firefly luciferase activity was measured and normalized to Renilla luciferase, ${ }^{*} \mathrm{P}<0.05$. (D) HepG2 cells were treated with cisplatin $(2 \mu \mathrm{M})$ plus NCO, miR-193b or Mcl-1 siRNA $(50 \mathrm{pmol} / \mathrm{ml})$ for $48 \mathrm{~h}$, and cell viability was determined by MTT assay, ${ }^{*} \mathrm{P}<0.05$. (E) pcDNA3.1-Mcl-1 $(2 \mu \mathrm{g} / \mathrm{ml})$ abolished the suppression of Mcl-1 caused by miR-193b transfection. The expression of Mcl-1 was determined by $\mathrm{qPCR}$ and western blot analysis, $\mathrm{P}<0.05$. $\mathrm{HCC}$, hepatocellular carcinoma; NCO, negative control oligonucleotide; 3'-UTR, 3'-untranslated region.

levels of miR-193b in the HepG2 cells, and the overexpression of miR-193b was confirmed by qPCR (Fig. 2A). We subsequently used the MTT assay to examine the effect of miR-193b on cisplatin treatment in HepG2 cells. As shown in Fig. 2B, the enforced expression of miR-193b plus cisplatin led to a significant decrease in the viability of the HepG2 cells compared to the viability in the cells treated with cisplatin combined with NCO. Furthermore, we selected a moderate concentration of cisplatin $(2 \mu \mathrm{M})$ for the combination treatment with miR-193b to detect the apoptosis induction. As shown in Fig. $2 \mathrm{C}$, more apoptotic cells were observed in the group treated with the combination of cisplatin and miR-193b mimic than the single-treatment group. Together, these results indicated that
miR-193b efficiently sensitized the HepG2 cells to cisplatin cytotoxicity in vitro.

Mcl-1 is the direct target of miR-193b in HepG2 cells. In order to explore the molecular mechanisms responsible for the sensitization by miR-193b to cisplatin treatment, we used TargetScan database and found that Mcl-1 may be a putative target of miR-193b (Fig. 3A). We then showed that the transfection of miR-193b significantly suppressed the expression of Mcl-1 in the HepG2 cells (Fig. 3B). To validate whether Mcl-1 is an actual target of miR-193b, an Mcl-1 3'-UTR fragment containing either a wild-type or mutant miR-193b binding sequence (Fig. 3A) was cloned into the pMIR reporter 
A

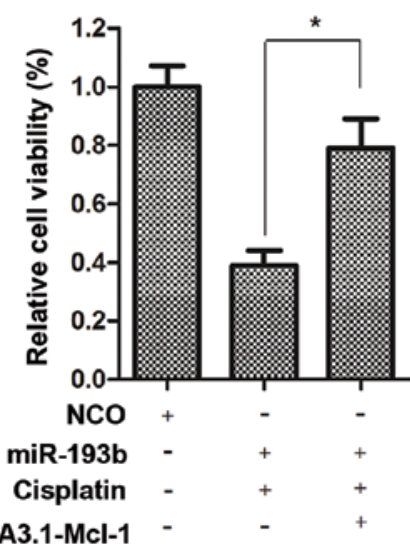

B

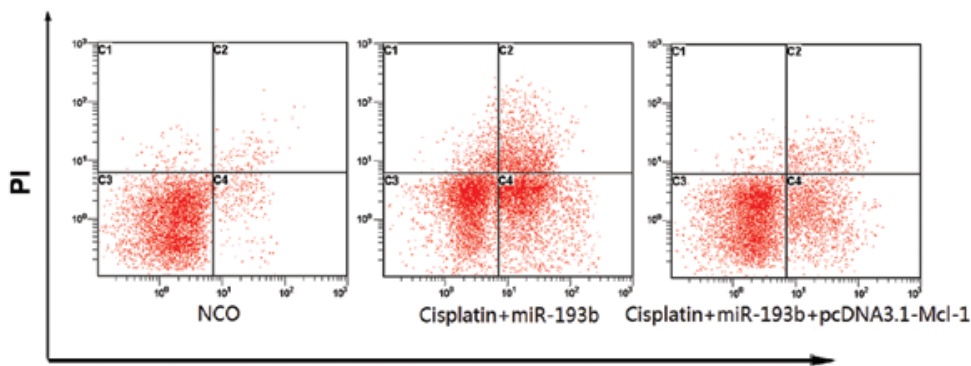

Annexin V-FITC

Figure 4. miR-193b functions as a cisplatin sensitizer via the direct targeting of Mcl-1. HepG2 cells were co-transfected with pcDNA3.1-Mcl-1 (2 $\mu \mathrm{g} / \mathrm{ml})$ and $\mathrm{miR}-193 \mathrm{~b}(50 \mathrm{pmol} / \mathrm{ml})$ for $24 \mathrm{~h}$. Subsequently, cisplatin $(2 \mu \mathrm{M})$ was added, and incubation was carried out for another $48 \mathrm{~h}$. (A) MTT assay was performed to measure the viability of the HepG2 cells, ${ }^{*} \mathrm{P}<0.05$. (B) The apoptotic rate was determined by Annexin V/PI staining and flow cytometry. HCC, hepatocellular carcinoma; PI, propidium iodide.

A

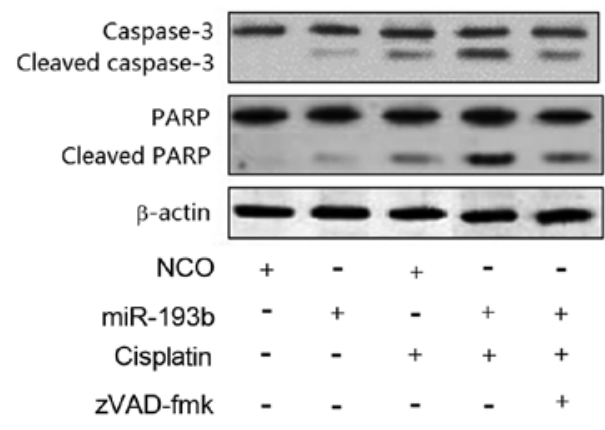

B

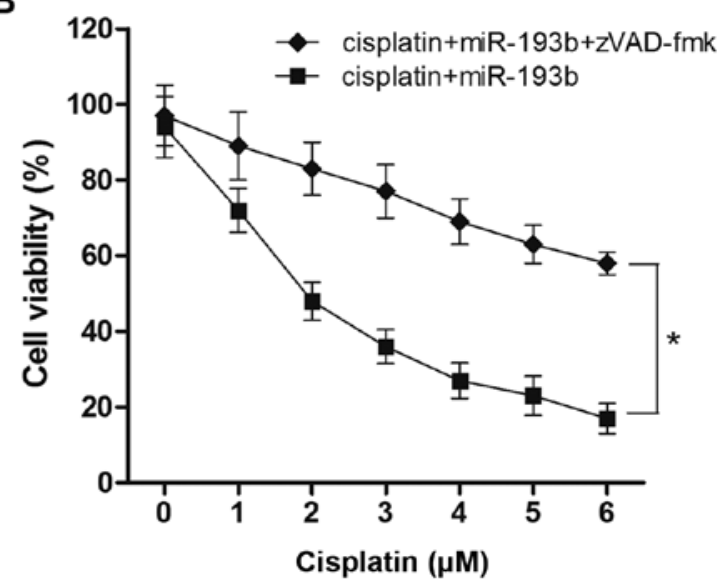

Figure 5. Activation of caspase-3 is essential for the sensitization by miR-193b to cisplatin-mediated cell death. (A) HepG2 cells were treated with cisplatin $(2 \mu \mathrm{M})$ plus miR-193b or NCO $(50 \mathrm{pmol} / \mathrm{ml})$ for $24 \mathrm{~h}$ in the absence or presence of zVAD-fmk $(10 \mu \mathrm{M})$. Western blot analysis was performed to measure the cleavage of caspase-3 and its substrate PARP. (B) HepG2 cells were treated with different concentrations of cisplatin plus miR-193b (50 pmol/ml) for $48 \mathrm{~h}$ in the absence or presence of zVAD-fmk $(10 \mu \mathrm{M})$. Cell viability was determined by MTT assay, ${ }^{*} \mathrm{P}<0.05$. NCO, negative control oligonucleotide; PARP, polyADP-ribose polymerase.

plasmid. After co-transfection with the pMIR reporter and miR-193b, a reduction in luciferase activity was observed for the wild-type construct-containing HepG2 cells. In contrast, the luciferase activity of the mutant or empty reporter in the presence of miR-193b was almost unaffected (Fig. 3C). More importantly, transfection of Mcl-1 siRNA exhibited a function similar to miR-193b on sensitizing HepG2 cells to cisplatin-inducing cytotoxicity (Fig. 3D). In addition, transfection with pcDNA3.1-Mcl-1 which contained no 3'-UTR sequences totally overcame the suppression of Mcl-1 caused by miR-193b (Fig. 3E). Taken together, these results suggest that the expression of Mcl-1 was negatively regulated by miR-193b in the HCC cells, which may play an essential role in the synergism of miR-193b with cisplatin treatment.

miR-193b sensitizes cisplatin-induced apoptosis by downregulation of the expression of Mcl-1. Our preceding results showed that miR-193b sensitized cisplatin-induced apoptosis in HepG2 cells and Mcl-1 is the direct target of miR-193b.
To further confirm whether miR-193b functions as a cisplatin sensitizer via the direct targeting of Mcl-1, HepG2 cells were co-transfected with pcDNA3.1-Mcl-1 and miR-193b for $24 \mathrm{~h}$. Then, the culture media were replaced with fresh medium and cisplatin was added, and incubation was carried out for another $48 \mathrm{~h}$. We found that the forced expression of Mcl-1 significantly decreased the growth inhibitory effect (Fig. 4A) and apoptotic rate (Fig. 4B) in the combined treatment of cisplatin plus miR-193b.

miR-193b increases cisplatin-induced cell death via the caspase-3-dependent pathway in HepG2 cells. Since the preceding results proved that the upregulation of miR-193b enhanced cell apoptosis caused by cisplatin, we investigated whether the synergistic effect of miR-193b on cisplatin-induced cell death is related with the caspase pathway. As shown in Fig. 5A, treatment with cisplatin plus miR-193b resulted in obvious cleavage of caspase- 3 and its substrate PARP, and the activation of caspase- 3 was impaired by the caspase inhibitor 
zVAD-fmk (15). In contrast, cisplatin treatment alone showed a weak activation of caspase-3 in the HepG2 cells (Fig. 5A). More importantly, cell death induced by cisplatin combined with miR-193b treatment was inhibited in the presence of zVAD-fmk, suggesting that the sensitization by miR-193b to cisplatin-mediated cell death was caspase-dependent (Fig. 5B).

\section{Discussion}

Studies have demonstrated that miR-193b acts as a tumor suppressor in multiple cancer types. Mu et al demonstrated that downregulation of miR-193b is significantly correlated with differentiation, invasion and metastasis in gastric cancer (16). $\mathrm{Li}$ et al showed that the expression of miR-193b was markedly decreased in pancreatic cancer and the transfection of miR-193b significantly inhibited the proliferation, invasion and metastasis of Panc-1 cells (17). In the present study, we showed that the expression of miR-193b was downregulated in liver tumors and HCC cell lines, suggesting that miR-193b is a tumor suppressor in HCC.

Previous research has confirmed that some specific miRNAs enhance the antitumor effect of chemotherapeutic drugs in multiple tumor types, including HCC (18-20). However, the molecular mechanisms of sensitization to chemotherapy caused by miRNAs remain unclear. Our findings demonstrated that miR-193b sensitized HepG2 cells to cisplatin-dependent cytotoxicity, inducing apoptosis.

Apoptosis is essential for normal development and homeostasis in a healthy body $(21,22)$. Aberrant regulation of apoptosis plays an essential role in multiple human diseases including cancer, inducing tumorigenesis and acquired chemotherapy resistance $(23,24)$. The apoptosis pathway is largely mediated by the Bcl-2 family proteins, which decide whether a cell continues to live or undergoes death through the intrinsic or mitochondrial apoptotic pathway $(25,26)$. Among them, Mcl-1 is one of the anti-apoptotic Bcl-2 family members, which plays a key role in apoptosis-resistance and is often overexpressed in human cancers (12). Recently, Mcl-1 has been confirmed to be regulated by miRNAs and was found to be associated with the curative effect of cisplatin in ovarian and breast cancer $(27,28)$. In the present study, we identified miR-193b as a direct regulator of Mcl-1. Notably, knockdown of the Mcl-1 gene by specific siRNA showed a function similar to miR-193b on sensitizing HepG2 cells to cisplatin, inducing cell death. In contrast, enforced expression of Mcl-1 by pcDNA3.1-Mcl-1 vector 'rescued' the HepG2 cells from apoptosis induced by cisplatin combined with miR-193b. These data emphasize the key role of Mcl-1 in cisplatin-dependent apoptosis of HCC cells.

Since the activation of caspase is a key event in the process of apoptosis, and the cleavage of caspase- 3 is a common step in both the extrinsic and apoptosis pathway (29), we further studied whether the synergistic effect of miR-193b on cisplatin-inducing cell death is caspase-3-dependent. According to our results, treatment with cisplatin plus miR-193b led to obvious cleavage of caspase- 3 and its substrate PARP, and zVAD-fmk increased the resistance of HepG2 cells to cisplatin plus miR-193b-induced cell death. We, therefore, conclude that miR-193b acts as a cisplatin sensitizer via the caspase-3-dependent pathway in HCC chemotherapy.
In summary, the present study demonstrated that miR$193 \mathrm{~b}$ is downregulated in human liver cancer cells. Moreover, miR-193b increased the sensitivity of HepG2 cells to the chemotherapy agent cisplatin by promoting apoptosis. Importantly, Mcl-1 was confirmed as a target of miR-193b in HCC, and the overexpression of miR-193b enhanced the sensitivity of cancer cells through the caspase-dependent apoptosis pathway. Our data demonstrated an important role for miR-193b in cisplatin therapy, which may provide a novel therapeutic strategy for the treatment of HCC.

\section{Acknowledgements}

The present study was supported by the National Natural Science Foundation of China (grant no. 81172575), the Hunan Provincial Natural Science Committee and Hengyang City Government Unification Foundation of China (grant no. 12JJ9033) and the Hunan Provincial Natural Science Foundation of China (grant no. 13JJ3079).

\section{References}

1. Jemal A, Bray F, Center MM, Ferlay J, Ward E and Forman D: Global cancer statistics. CA Cancer J Clin 61: 69-90, 2011.

2. Nault JC, Calderaro J, Di Tommaso L, Balabaud C, Zafrani ES, Bioulac-Sage P, Roncalli M and Zucman-Rossi J: Telomerase reverse transcriptase promoter mutation is an early somatic genetic alteration in the transformation of premalignant nodules in hepatocellular carcinoma on cirrhosis. Hepatology 60: 1983-1992, 2014.

3. Kerr SH and Kerr DJ: Novel treatments for hepatocellular cancer. Cancer Lett 286: 114-120, 2009.

4. Arslan C, Ozdemir E, Dogan E, Ozisik Y and Altundag K: Secondary hematological malignancies after treatment of non-metastatic breast cancer. J BUON 16: 744-750, 2011.

5. Roufayel R, Johnston DS and Mosser DD: The elimination of miR-23a in heat-stressed cells promotes NOXA-induced cell death and is prevented by HSP70. Cell Death Dis 27: e1546, 2014.

6. Nair N and Gongora E: MicroRNAs as therapeutic targets in cardiomyopathies: Myth or reality? Biomol Concepts 5: 439-448, 2014.

7. Zhang H, Cheng Y, Jia C, Yu S, Xiao Y and Chen J: MicroRNA-29s could target AKT2 to inhibit gastric cancer cells invasion ability. Med Oncol 32: 342, 2015.

8. Zhang L, Ge Y and Fuchs E: miR-125b can enhance skin tumor initiation and promote malignant progression by repressing differentiation and prolonging cell survival. Genes Dev 28: 2532-2546, 2014

9. Wang S and Li K: MicroRNA-96 regulates RGC-5 cell growth through caspase-dependent apoptosis. Int J Clin Exp Med 7: 3694-3702, 2014.

10. Wolfson B, Eades G and Zhou Q: Roles of microRNA-140 in stem cell-associated early stage breast cancer. World J Stem Cells 6: 591-597, 2014.

11. Mo ZH, Wu XD, Li S, Fei BY and Zhang B: Expression and clinical significance of microRNA-376a in colorectal cancer. Asian Pac J Cancer Prev 15: 9523-9527, 2014.

12. Woo SM, Min KJ, Seo BR, Nam JO, Choi KS, Yoo YH and Kwon TK: Cafestol overcomes ABT-737 resistance in Mcl-1-overexpressed renal carcinoma Caki cells through downregulation of Mcl-1 expression and upregulation of Bim expression. Cell Death Dis 5: e1514, 2014.

13. Chen C, Ridzon DA, Broomer AJ, Zhou Z, Lee DH, Nguyen JT, Barbisin M, Xu NL, Mahuvakar VR, Andersen MR, et al: Real-time quantification of microRNAs by stem-loop RT-PCR. Nucleic Acids Res 33: e179, 2005.

14. Livak KJ and Schmittgen TD: Analysis of relative gene expression data using real-time quantitative PCR and the 2(-Delta Delta C(T)) method. Methods 25: 402-408, 2001.

15. Amaral C, Borges M, Melo S, da Silva ET, Correia-da-Silva G and Teixeira N: Apoptosis and autophagy in breast cancer cells following exemestane treatment. PLoS One 7: e42398, 2012. 
16. Mu YP, Tang S, Sun WJ, Gao WM, Wang M and Su XL: Association of miR-193b down-regulation and miR-196a up-regulation with clinicopathological features and prognosis in gastric cancer. Asian Pac J Cancer Prev 15: 8893-8900, 2014.

17. Li J, Kong F, Wu K, Song K, He J and Sun W: miR-193b directly targets STMN1 and uPA genes and suppresses tumor growth and metastasis in pancreatic cancer. Mol Med Rep 10: 2613-2620, 2014.

18. Liu R, Liu X, Zheng Y, Gu J, Xiong S, Jiang P, Jiang X, Huang E, Yang Y, Ge D, et al: MicroRNA-7 sensitizes non-small cell lung cancer cells to paclitaxel. Oncol Lett 8: 2193-2200, 2014.

19. Boyerinas B, Park SM, Murmann AE, Gwin K, Montag AG, Zillhardt M, Hua YJ, Lengyel E and Peter ME: Let-7 modulates acquired resistance of ovarian cancer to Taxanes via IMP-1-mediated stabilization of multidrug resistance 1. Int J Cancer 130: 1787-1797, 2012.

20. Koga C, Kobayashi S, Nagano H, Tomimaru Y, Hama N, Wada H, Kawamoto K, Eguchi H, Konno M, Ishii H, et al: Reprogramming using microRNA-302 improves drug sensitivity in hepatocellular carcinoma cells. Ann Surg Oncol 21 (Suppl 4): S591-S600, 2014

21. Creagh EM: Caspase crosstalk: integration of apoptotic and innate immune signalling pathways. Trends Immunol 35: 631-640, 2014

22. Childs BG, BakerDJ, Kirkland JL, Campisi J and van Deursen JM: Senescence and apoptosis: Dueling or complementary cell fates? EMBO Rep 15: 1139-1153, 2014.
23. Setia S, Nehru B and Sanyal SN: Celecoxib prevents colitis associated colon carcinogenesis: An upregulation of apoptosis. Pharmacol Rep 66: 1083-1091, 2014.

24. Yang P, Tuo L, Wu Q and Cao X: Licochalcone-A sensitizes human esophageal carcinoma cells to TRAIL-mediated apoptosis by proteasomal degradation of XIAP. Hepatogastroenterology 61: 1229-1234, 2014.

25. Lee HG, Lee JM, Shin SJ, Kwon SH, Lee GS, Song CH, Choi ES, Cha SD and Cho CH: Salinomycin inhibited cell proliferation and induced apoptosis in human uterine leiomyoma cells. Obstet Gynecol Sci 57: 501-506, 2014.

26. Zhao J, Li X, Zou M, He J, Han Y, Wu D, Yang H and Wu J: miR-135a inhibition protects A549 cells from LPS-induced apoptosis by targeting Bcl-2. Biochem Biophys Res Commun 452: 951-957, 2014.

27. Rao YM, Shi HR, Ji M and Chen CH: miR-106a targets Mcl-1 to suppress cisplatin resistance of ovarian cancer A2780 cells. J Huazhong Univ Sci Technolog Med Sci 33: 567-572, 2013.

28. Zhang R, Li Y, Dong X, Peng L and Nie X: miR-363 sensitizes cisplatin-induced apoptosis targeting in Mcl-1 in breast cancer. Med Oncol 31: 347, 2014.

29. Geserick P, Wang J, Feoktistova $M$ and Leverkus M: The ratio of Mcl-1 and Noxa determines ABT737 resistance in squamous cell carcinoma of the skin. Cell Death Dis 5: e1412, 2014. 